\title{
The distribution of events in the human menstrual cycle
}

\author{
J. R. Udry and Naomi M. Morris \\ Department of Maternal and Child Health, School of Public Health, \\ University of North Carolina, Chapel Hill, North Carolina 27514, U.S.A.
}

\begin{abstract}
Summary. Daily reports of 85 married couples concerning their sexual behaviour for about 3 menstrual cycles per couple were organized according to menstrual events by using six techniques of aggregation. While there were some similarities among the different displays, including an apparent peak about 6 days before mid-cycle, different methods of aggregation produce widely different frequency curves. It is concluded that there is no single method of display of events of the menstrual cycle which will fit all investigations.
\end{abstract}

\section{Introduction}

It has long been presumed that the behaviour of women is correlated with the rhythm of the menstrual cycle. Most writers on the subject have related the presumption to a theory of hormonal effects on behaviour. An association of violence and behavioural disorders with the premenstrual days has been proposed (Ellis \& Austin, 1971) and sexual desire and sexual behaviour have been associated with menstrual rhythms (Udry \& Morris, 1968). Other emotional states have been shown to vary according to menstrual patterns (Moos et al., 1969). The ease of identifying the beginning and end of particular cycles according to the onset of menses has led to the use of the menstrual marker as the orientation point around which the behaviour of interest was organized. If all women had the same length of menstrual cycle, with no inter- or intrapersonal variance, and if hormonal events could be presumed to proceed in an invariant pattern during every cycle, the menstrual marker would be an adequate reference point for the association of behavioural observations and hormonal events. There is, however, no such regularity. Not only is the average length of the cycle different for different women, but the intrapersonal variance has been found to be far higher than early research supposed (Treloar, Boynton, Behn \& Brown, 1967). Investigators have therefore been faced with the problem of how to organize data from cycles of disparate length. The particular strategy adopted has depended on the theoretical assumptions about the procession of hormonal events in cycles. Assuming that the pace of hormonal events is directly proportional to the cycle length, an arbitrary length of cycle (usually 28 days) can be taken and used as a standard; the events of longer cycles are squeezed together while events of shorter cycles are spread out in the cycle proportionate to the cycle length (Farris, 1956). Other workers have concluded that irregularities in the length of the cycle are associated with events before ovulation and that once ovulation occurs all cycles proceed at the same pace (Hertig, 1965). For this assumption, the data have to be organized by numbering days backwards from the onset of menses towards the middle of the cycle (i.e. reverse cycle days), and letting the early part of the cycle vary as it would. Still others have argued that hormonal events only in cycles of a given length could be compared, leading to the separate examination of cycles of different length (James, 1971).

Those who are interested in events occurring near the ends of cycles can number the days positively and negatively from the onset of menses, with the presumption that the displacement of hormonal events would be least on the days of greatest interest, while days farthest from the marker, and therefore containing the most displacement of events with respect to hormones, are not of particular interest. An interest in the organization of events in mid-cycle, i.e. at the presumed time of ovulation, is disadvantageous, because the behaviour of interest is farthest from the menstrual marker. The procedure of aggregating cycles of only the same length seems simple and sound, and no doubt would be more generally adopted, except for the fact that an enormous amount of data is required to obtain stable patterns for cycles of a particular length. 
The problem would be solved if there were adequate measures of the occurrence of hormonal events independent of the occurrence of menses. There was no practical way of doing this before the advent of radioimmunoassay techniques, except for the microscopic examination of vaginal squamous cells which could be used to identify the time of appearance of progesterone (Benedek, 1939). However, even with radioimmunoassay techniques the problem is far from solved. Many of the hormonal events of the cycle are very short in duration. In animals, indwelling cannulae can be inserted so that frequent blood samples can be withdrawn, but this approach is not practicable for obtaining daily blood samples from large groups of healthy women.

We have examined the patterns of distribution of sexual behaviour during the menstrual cycle of women by collecting daily reports of behaviour to avoid the bias of retrospective data collection. In our first study we organized the data by relating them to reverse menstrual cycle days (Udry \& Morris, 1968), but on the basis of papers by others we used standardized cycle days in our second study (Udry \& Morris, 1970). In both studies we found a mid-cycle peak and a luteal trough in sexual activity, a pattern which is altered by the administration of contraceptive pills (Udry, Morris \& Waller, 1973).

However, because of the variability of reference points and of data handling, the results of studies on the human menstrual cycle are difficult to compare. In the present study we have examined the same behaviour as in the previous papers, but have presented data analysed by different techniques to reveal the variation in results which can be obtained.

\section{Materials and Methods}

The sample consisted of 85 young married couples who were recruited largely from a university campus. Husbands and wives were asked to mail independent daily reports of their sexual behaviour and answers to related questions. Wives recorded their basal body temperatures. Daily mid-cycle blood samples were contributed by 43 women.

The information received was plotted for the wife's report of coitus, orgasm and whether she wanted intercourse. Six aggregating techniques were used, each with two different inclusion criteria. (1) Forward cycle days (FCD): the days are numbered forward from the date of onset of menstruation and the record stopped at 28 days. (2) Reverse cycle days (RCD) : these days are numbered backward from onset of menstruation until the 28th day. (3) Standardized cycle days (SCD): the events of the cycle are squeezed or expanded to fit a 28 -day cycle according to the formula, $S C D=(R C D \times 28)$ / $(\mathrm{RCD}+\mathrm{FCD})+1$, with the SCD value rounded to the nearest integer. (4) Spitz-Gold-Adams (SGA) method of standardizing: a grid identifies which days of which cycle length are to be assigned to each of seven cycle phases (Spitz, Gold \& Adams, 1975). (5) Temperature cycle days: for the $40 \%$ of cycles in which our fertility consultant could identify a specific basal body temperature nadir in the cycle, the nadir was called Day 0, and other days were numbered negatively and positively around that point. The graph was cut at +12 and -14 days. (6) Luteinizing hormone ( $\mathrm{LH}$ ) days: for the 34 cycles in which we were able to identify the day on which the LH surge occurred, the day of the surge was Day 0 , and other days were numbered negatively and positively around that point. The graph was cut at +12 and -14 days.

For each of these aggregating techniques, the results are plotted (a) for the maximum number of observations possible and therefore the cycles included in each panel are not necessarily the same cycles, and (b) for only the 27 cycles for which data were available for all six techniques.

\section{Results}

Intercourse. In Text-fig. 1(a), large numbers of cycles, there is a high area of the cycle about FCD 9, which is not especially striking in magnitude, in the FCD, RCD and SCD panels, with little of interest at mid-cycle or in the luteal phase. SGA shows a slight elevation in Phase III. These four all show 

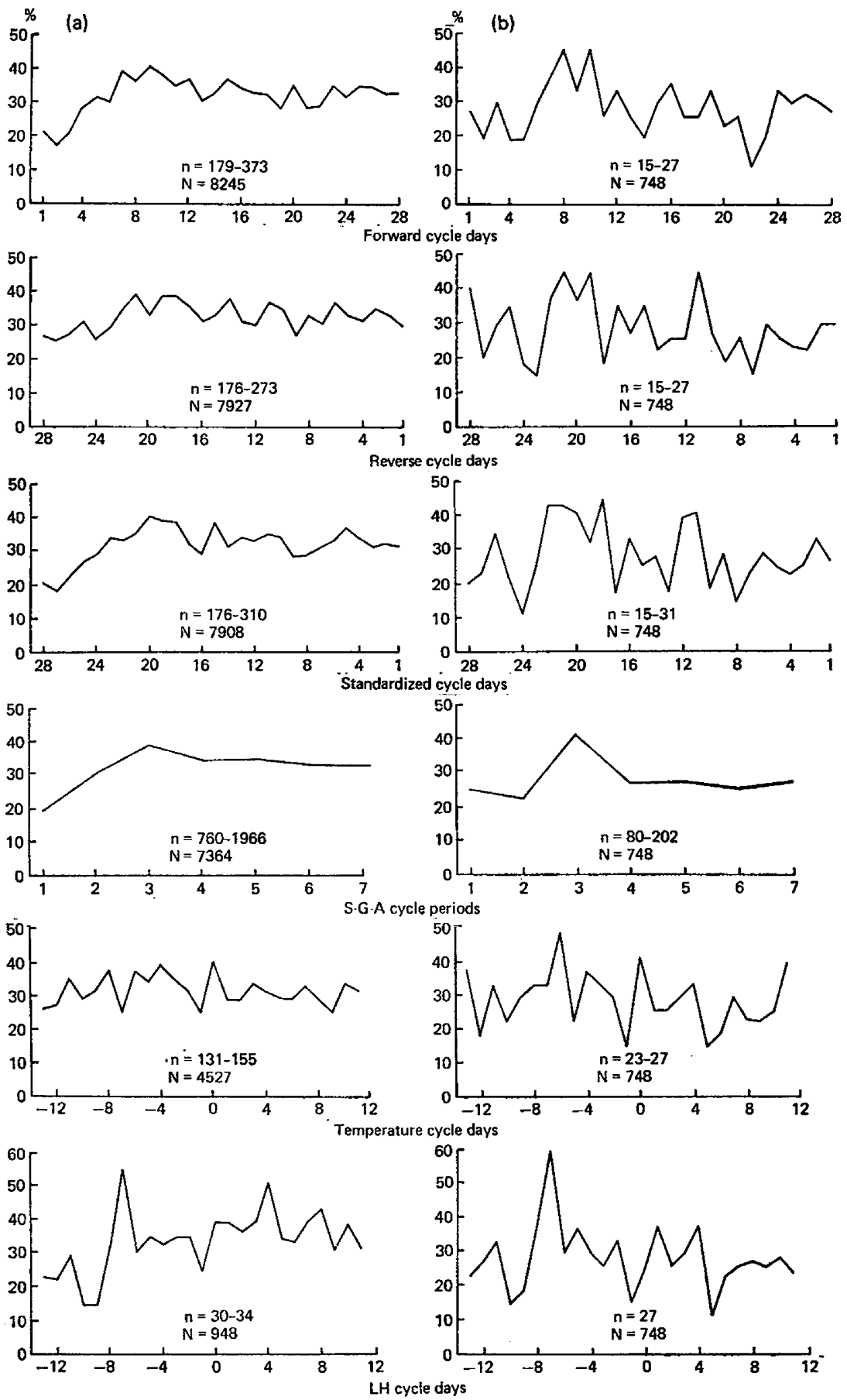

Text-fig. 1. The percentages of women reporting intercourse for each day of the menstrual cycle plotted according to different methods of standardizing data (see text) from (a) all available data for each method and (b) cycles for which data were available for all 6 aggregation techniques. $N=$ the number of events for each panel; $\mathrm{n}=$ the number of events at each point. 

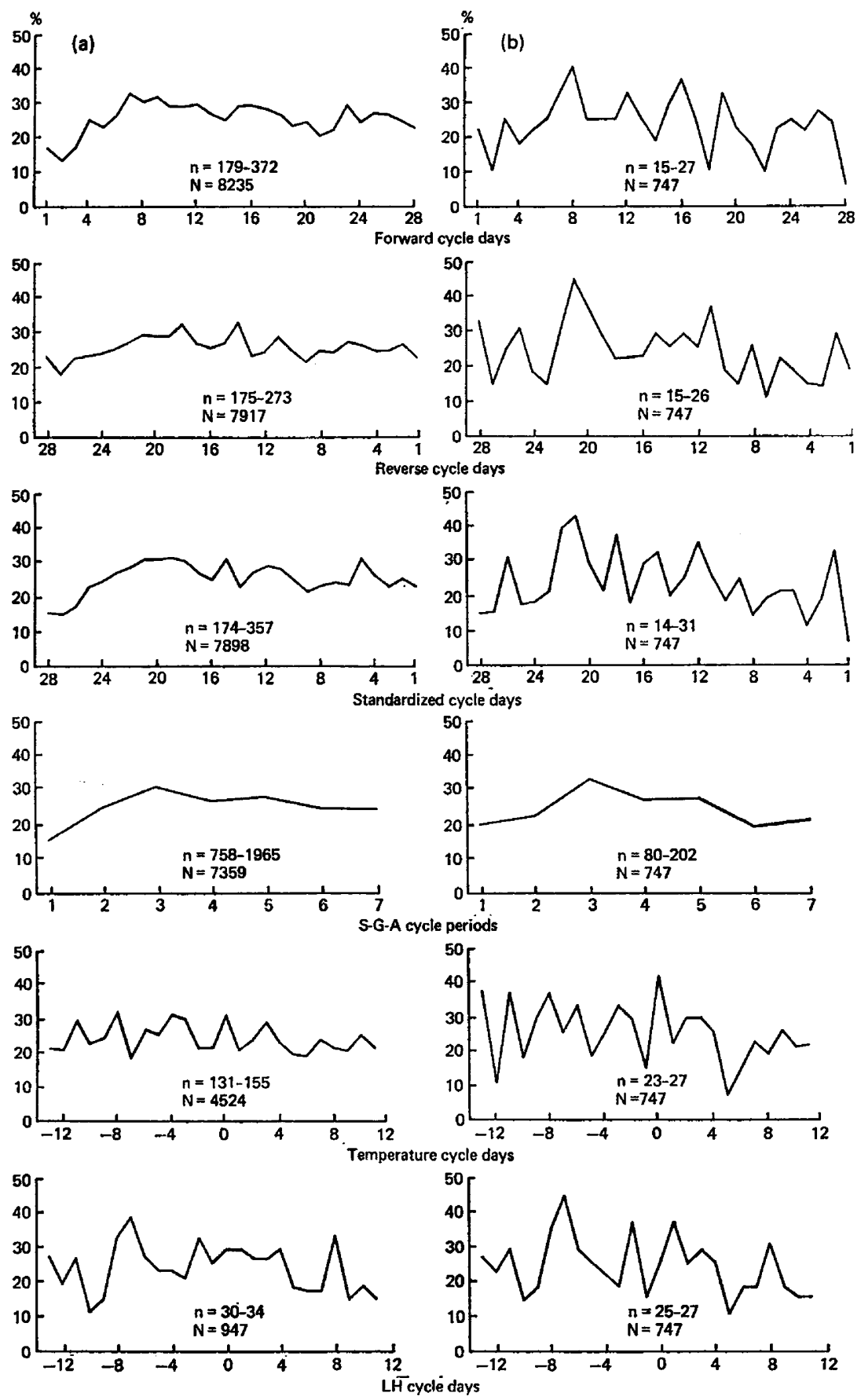

Text-fig. 2. The percentages of women reporting orgasm for each day of the menstrual cycle plotted according to different methods of standardizing data (see text) from (a) all available data for each method and (b) cycles for which data were available for all 6 aggregation techniques, $\mathrm{N}=$ the number of events for each panel; $\mathrm{n}=$ the number of events at each point. 

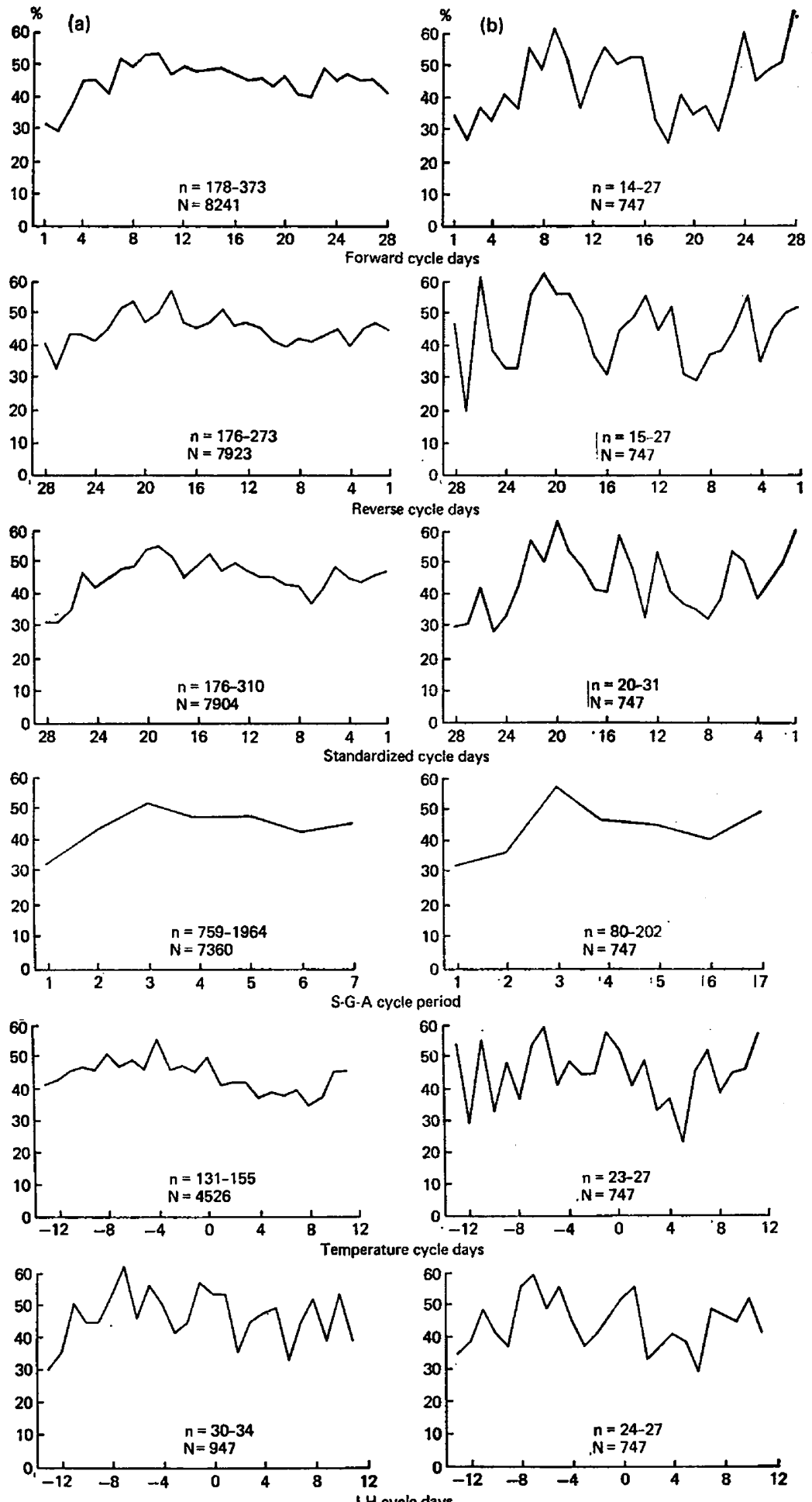

Text-fig. 3. The percentages of women reporting the desire for intercourse for each day of the menstrual cycle plotted according to different methods of standardizing data (see text) from (a) all available data for each method and (b) cycles for which data were available for all 6 aggregation techniques. $\mathrm{N}=$ the number of events for each panel; $n=$ the number of events at each point. 
depressions associated with menstrual flow. TCD shows low points at Days $-7,-1$ and +9 , with a high point on Day 0 . LHD shows a sharp spike on Day -7 , and a lesser spike on Day +4 .

The small numbers in Text-fig. 1(b), constant N, generate very unstable graphs. Many of the features seen in Text-fig. 1(a) are retained, but it is clear that the women in Text-fig. 1(b) are a biased sample of those in Text-fig. 1(a).

Orgasm. The results in Text-fig. 2(a) include orgasm not related to intercourse and show less clear patterns than those of Text-fig. 1(a).

The smaller numbers in Text-fig. 2(b) demonstrate quite dramatic orgasm patterns, with all but TCD showing a high point about 6 days before mid-cycle and a low period midway between midcycle and the end of the cycle. These patterns cannot be seen on Text-fig. 2(a), and must therefore be associated with peculiarities of the women in the smallest group.

Desire. In all the panels in Text-fig. 3(a) there is a consistent high area a few days before mid-cycle, and a decline to a low a week or more after mid-cycle. In Text-fig. 3(b) there is an exaggeration of the patterns shown in Text-fig. 3(a) on all graphs.

\section{General interpretation}

Looked at as a group, certain features of these results deserve attention. The high point on most of the graphs falls about 6 days before mid-cycle, which is consistent with the pioneering observations of McCance, Luff \& Widdowson (1937), and is different from the observations reported in our previous samples (Udry \& Morris, 1968, 1970), however the data were organized. It is also similar to some of the observations organized by James (1971) according to cycles of particular durations. The luteal trough previously observed in our samples appears on most of the arrangements of data. We now conclude, therefore, that different samples of women show different patterns of sexual behaviour in the menstrual cycle.

There is some indication that the sub-sample of women for whom we have data for all arrangements of the cycle show more distinct patterns than the entire sample. While the small sample size produces more erratic curves, the pattern is not lost, but exaggerated.

It is also evident that for some behaviour one method of displaying the data produces the most distinct patterns, while for other behaviour another method is distinctive. The TCD panel in Text-fig. 1(a) shows a distinct low point on Day -1 and a distinct high point for the cycle on Day 0 , with no noticeable other pattern. Since Day 0 is the putative day of ovulation, this data display would appear to be the choice for events presumably related to ovulation. On the other hand, we were only able to locate a temperature nadir in about $40 \%$ of the cycles, which no doubt biases the sample.

The orgasm graph from TCD (Text-fig. 2), particularly with the small N, shows a similar peak associated with ovulation. On the other hand, the LHD graphs, which should give an even better focus on ovulation, do not show the same pattern as the TCD. Why this should be is not at all clear.

Purposive selection from among the six displays for each behaviour, ignoring contradictory graphs, would allow the support of nearly any conclusion one would wish to reach. Therefore, in order for the investigator to protect himself from capitalizing on fortuitous random factors, it is necessary for him to select in advance, on theoretical grounds, the single display technique which most nearly fits his theoretical framework. It is clear from the inconsistent results of the many published investigations that comparability among studies cannot be obtained by the use of noncompatible data displays.

In the future, we can only support the use of the menstrual marker for influences on sexual behaviour believed to be directly related to menstruation. The only such relationship is the differential tendency of individuals to avoid intercourse during menstruation. Even in this particular case, rather than arranging data according to the onset of menses, it is preferable simply to separate menstrual from non-menstrual days. With respect to the release from menstrual abstinence discussed by James (1971), the index point should obviously be the last day of menstruation, not the first.

We have probably gone about as far as we can go with data arranged according to menstrual markers. With the development of assay techniques for most steroids of interest, and with the elabora- 
tion of specific theories about the behavioural concommitants of serum steroid fluctuations, menstrual markers have become outdated for research in this area. The convenience and economy of the menstrual marker is not enough of an excuse to overcome its theoretical irrelevance to most theory about the relationship between hormones and patterns of sexual behaviour. Progress in the future in this area must come from the explication of a relationship between a particular hormone(s), or combination thereof, and a particular kind of sexual behaviour. Direct assay of serum fluctuations in the substance of interest and correlation with the sexual behaviour of intercst is then the research design of choice.

This research was supported by University Research Council Grant No. 1-0-160-4645-VF254, General Research Support Grant No. 5-S01-RR-05450-12, and NICHD Grant No. 1-R01HD07454-01.

\section{References}

BENEDeK, T. (1939) The correlations between ovarian activity and psychodynamic processes I. The ovulation phase. Psychosom. Med. 1, 245-270.

Ellis, D.P. \& AustiN, P. (1971) Menstruation and aggressive behaviour in a correctional center for women. J. Crim. Law Criminol. \& Police Science 3, 388-395.

FARRIS, E.J. (1956) Human Ovulation and Fertility. Lippincott, Philadelphia.

Hertig, A.T. (1965) Morphologic criteria of the time of ovulation in the human being. In Human Ovulation, pp. 75-83. Ed. C. S. Keefer. Little, Brown and Company, Boston.

JAMES, W.H. (1971) The distribution of coitus within the human intermenstruum. J. biosoc. Sci. 3, 159171.

MCCANCE, R.A., LuFf, M.C. \& Widdowson, E.E. (1937) Physical and emotional periodicity in women. J. Hyg., Camb. 37, 571-611.

Moos, R.H., Kopell, B.S., Melges, F.T., Yalom, I.,
Lunde, D., Clayton, R. \& Hamburg, E., (1969) Variations in symptoms and mood during the menstrual cycle. J. Psychosom. Res. 13, 37-44.

SpITz, C.J., Gold, A.R. \& ADAMs, D.B. (1975) Cognitive and hormonal factors affecting coital frequency. Arch. Sex. Behav. 4, 249-263.

Treloar, A.E., Boynton, R.E., Behn, B.G. \& Brown, B.W. (1967) Variation of the human menstrual cycle through reproductive life. Int. J. Fert. 1, 77-126.

UDRY, J.R. \& MORRIS, N.M. (1968) Distribution of coitus in the menstrual cycle. Nature, Lond. 220, 593-596.

UDRY, J.R. \& MORRIS, N.M. (1970) The effect of contraceptive pills on the distribution of sexual activity in the menstrual cycle. Nature, Lond. 227, 502-503.

UDRY, J.R., MORRIS, N.M. \& WALler, L. (1973) Effect of contraceptive pills on sexual activity in the luteal phase of the human menstrual cycle. Arch. Sex. Behav. 2, 207-214. 